\title{
Anterograde Transport and Secretion of Brain-Derived Neurotrophic Factor along Sensory Axons Promote Schwann Cell Myelination
}

\author{
Benjamin K. Ng, ${ }^{1 *}$ Lian Chen, ${ }^{2 *}$ Wilhelm Mandemakers, ${ }^{3}$ José M. Cosgaya, ${ }^{4}$ and Jonah R. Chan ${ }^{1}$ \\ ${ }^{1}$ Zilkha Neurogenetic Institute, Keck School of Medicine, University of Southern California, Los Angeles, California 90033, ${ }^{2}$ National Institute of Child \\ Health and Development, National Institutes of Health, Bethesda, Maryland 20892, ${ }^{3}$ Department of Human Genetics, Katholieke Universiteit Leuven, 3000 \\ Leuven, Belgium, and ${ }^{4}$ Instituto de Investigaciones Biomedicas, Consejo Superior de Investigaciones, Cientificas and Universidad Autonoma de Madrid, \\ 28029 Madrid, Spain
}

\begin{abstract}
The neurotrophin brain-derived neurotrophic factor (BDNF) inhibits Schwann cell (SC) migration and promotes myelination via the p75 neurotrophin receptor (NTR). Despite these recent findings, the expression, localization, and mechanism of BDNF action has yet to be determined. Here we demonstrate that the sensory neurons of the dorsal root ganglion (DRG) are a major source of BDNF during postnatal development. The expression of BDNF is initially elevated before myelination and decreases dramatically after the onset of myelination. BDNF expression is controlled in part by transcriptional regulation and the increased expression of the truncated TrkB receptor on SCs. To investigate the possible mechanism of BDNF transport and release, multicompartment Campenot chambers were used. DRG neurons transported and secreted endogenous BDNF along the surface of axons in anterograde fashion. In an attempt to enhance myelination by SCs, DRG neurons were transduced with an adenovirus to overexpress BDNF. BDNF was transported and secreted along the axons and enhanced myelination when compared with control cocultures. Together, the events surrounding the expression, localization, and mechanism of BDNF action in DRG neurons may hint at potential therapeutic implications to efficiently promote remyelination.
\end{abstract}

Key words: Schwann cell; myelination; dorsal root ganglion neurons; neurotrophins; anterograde; BDNF

\section{Introduction}

The complex interplay between glia and neurons is essential for the development and function of the nervous system. Schwann cell (SC) proliferation, migration, and myelination are undoubtedly controlled by signals that are directed from the neuron. Neurotrophins, a family of growth factors initially found to be critical for neuronal survival and outgrowth (Huang and Reichardt, 2001), have been implicated recently as key factors involved in SC development (Hempstead, 2005). Specifically, brain-derived neurotrophic factor (BDNF) was shown to enhance myelination in SCs through p75 ${ }^{\text {NTR }}$ and to subsequently inhibit migration through the RhoA/Rho kinase pathway (Cosgaya et al., 2002; Yamauchi et al., 2004). On the contrary, neurotrophin 3 (NT3)

Received Feb. 8, 2007; revised May 9, 2007; accepted June 5, 2007.

This work was supported by grants from the Ministerio de Educacion y Ciencia, Comunidad de Madrid, and Fundacion Mutua Madrileña (J.M.C.) and the National Multiple Sclerosis Society Career Transition Award and the Donald E. and Delia B. Baxter Foundation Award (J.R.C.). We acknowledge Dr. E. M. Shooter for his support, encouragement, and scientific insight; Dr. A. Kruttgen for establishing the BDNF ELISA; Dr. Y. J. Wu for the adenovirus constructs; and Dr. E. E. Kelland, S. S. Rosenberg, and S. Y. Chong for critically reading this manuscript and for insightful discussion.

${ }^{*}$ B.K.N. and L.C. contributed equally to this work.

Correspondence should be addressed to Dr. Jonah R. Chan, Zilkha Neurogenetic Institute, Department of Biochemistry and Molecular Biology, Keck School of Medicine, University of Southern California, 1501 San Pablo Street, ZNI 421, Los Angeles, CA 90033. E-mail: jonah.chan@usc.edu.

D01:10.1523/JNEUROSCI.0563-07.2007

Copyright $\odot 2007$ Society for Neuroscience $\quad$ 0270-6474/07/277597-07\$15.00/0 signaling in SCs enhanced cell migration and inhibited myelin formation via the full-length TrkC receptor (Cosgaya et al., 2002; Yamauchi et al., 2003). The temporal relationship between NT3 and BDNF signaling was examined in vitro. NT3 levels were elevated during SC proliferation and migration and decreased during myelination, whereas BDNF levels remained elevated throughout (Cosgaya et al., 2002). The results of these experiments have begun to shed light on the important yet distinct roles that neurotrophins play during peripheral nerve development.

Although the function of BDNF during myelination is becoming more apparent, it is still not clear which cell type produces BDNF or how it is being transported. If the expression of BDNF can be localized during myelination, will focal overexpression augment the myelination process and provide a more efficient therapeutic approach in cases of demyelination? Recent evidence points to the expression of BDNF in neurons and secretion at nerve terminals that are most likely in contact with target tissues (Barakat-Walter, 1996; Tonra, 1999; Lessmann et al., 2003). This suggests that BDNF is transported in anterograde fashion. However, because SCs do not myelinate nerve terminals, could BDNF also be secreted along the axon? Additionally, it is also quite possible that BDNF is secreted by SCs, activating $\mathrm{p} 75^{\mathrm{NTR}}$ in an autocrine manner (Friedman et al., 1999; Esper and Loeb, 2004). Here we demonstrate that sensory neurons of the dorsal root ganglion (DRG) are a major source of BDNF during postnatal develop- 
ment when compared with SCs. DRG neurons secrete endogenous BDNF along their axons in anterograde fashion to promote SC myelination. The expression and secretion of BDNF by DRG neurons is dynamically regulated at both the transcriptional level and by the induction of the truncated TrkB/T1 receptor. Additionally, adenoviral-mediated overexpression of BDNF in DRG neurons significantly enhances myelination by SCs in coculture. The overexpression and presentation of BDNF as outlined in this study may give rise to future experiments that assess whether remyelination can be achieved through the localized delivery of BDNF after demyelination attributable to injury or disease.

\section{Materials and Methods}

Primary cell culture. Purified DRG neurons and SCs were established using methods described by Kleitman et al. (1998) and Chan et al. (2004). Briefly, DRG neurons from embryonic day 15 Sprague Dawley rats were dissociated, plated, and purified on collagen-coated coverslips in the presence of NGF $(100 \mathrm{ng} / \mathrm{ml})$. Neurons were purified before addition of SCs. Myelination was initiated with the addition of ascorbic acid (50 $\mu \mathrm{g} / \mathrm{ml})$.

Campenot chambers. The compartmentalized Campenot chambers were prepared as described previously (Campenot, 1992; Chan et al., 2004). DRG neurons were plated into the side compartment of the chamber, and axons were allowed to grow into the second and third compartment in the presence of NGF. Antimitotic medium was used to eliminate non-neuronal cells. The integrity of the seal between compartments was tested quantitatively throughout the experiment by monitoring the ability of phenol red to diffuse to phenol red-free compartments.

Adenovirus production and infection of DRG neurons. cDNA for the myc-tagged BDNF was subcloned into pAdTrackCMV for the generation of recombinant adenovirus (pAdEasy system) (He et al., 1998; Wu et al., 2004). myc-BDNF was subcloned with SalI and XbaI, and large-scale virus preparations were performed in human embryonic kidney HEK293 cells using the cesium chloride banding method. Adenovirus titers were determined as described previously (Graham and Prevec, 1995). Subsequently, purified recombinant adenovirus was added directly to the neurons at a multiplicity of infection of 10 .

Competitive reverse transcription-PCR. Competitive reverse transcription (RT)-PCR was performed for the ribosomal protein L19 (internal control) and BDNF using methods as described by Friedman et al. (1996). The competitors for L19 and BDNF were PCR amplified from a rat sciatic nerve cDNA library with an internal $30 \mathrm{nt}$ deletion and cloned into the pGEM-T vector using the QuickChange Site-Directed Mutagenesis kit (Stratagene, La Jolla, CA). The RNA from sciatic nerves, spinal cords, and cocultures were extracted using the TRIZOL reagent (Invitrogen, Carlsbad, CA). Reverse transcription was performed with $8 \mu \mathrm{g}$ of total RNA after DNase I (GE Healthcare, Little Chalfont, UK) treatment. The PCR reactions included $40 \mathrm{ng}$ of RNA from the RT reactions for the different samples and a series of dilutions for the L19 and BDNF competitors. Each sample consisted of at least seven different concentrations of competitor. Quantification was achieved using the NIH Image 1.62 software and by performing a linear regression of log (competitor/gene of interest) with $\log$ (concentration of the competitor). The point at which $\log$ (competitor/gene of interest) is zero is the equivalence point; the antilog gives the concentration of the gene of interest. The SE of estimate was calculated from the regression model of each sample.

BDNF ELISA. ELISAs were performed using the TMB (tetramethylbenzidine) Peroxidase Substrate System (KPL, Gaithersburg, MD), as described previously (Kruttgen et al., 1998; Chan et al., 2001). Briefly, the BDNF ELISA was accomplished using the TrkB-Fc fusion protein (Regeneron Pharmaceuticals, Tarrytown, NY) as the capturing reagent, followed by incubation of the samples. The BDNF was detected using a polyclonal chicken anti-BDNF primary antibody followed with an antichicken HRP antibody (Promega, Madison, WI). The substrate was incubated for $\sim 2-5$ min or until adequate signal was detected. ELISA reactions were stopped with the addition of $1 \mathrm{~m}$ phosphoric acid.

Western blot analysis. Samples from primary cultures were prepared for Western blot as described previously (Chan et al., 2004). The proteins were transferred to pure nitrocellulose membranes and probed with specific antibodies. p75 ${ }^{\mathrm{NTR}}$, TrkB/T1, and TrkA were probed with rabbit antibodies against each specific neurotrophin receptor [anti-p75 ${ }^{\text {NTR }}$ (Promega), anti-TrkB (Transduction Laboratories, Lexington, KY), and anti-TrkA (gift from Louis F. Reichardt, University of California, San Francisco, CA)]. Mouse antibodies against myelin associated glycoprotein (MAG) and $\mathrm{P}_{0}$, were used to identify the extent of myelination in the cultures [anti-MAG (Chemicon, Temecula, CA) and anti- $\mathrm{P}_{0}$ (from Juan J. Archelos, Karl-Franzen-Universitat, Graz, Austria)]. Additionally, an antibody against $\beta$-actin (Sigma, St. Louis, MO) was used as a loading control. Blots were typically stripped and reprobed.

Immunostaining. Immunostaining of SC/DRG cocultures was performed as described previously (Chan et al., 2004). Briefly, cultures were washed and fixed using $4 \%$ paraformaldehyde and then permeabilized and blocked by incubation with $20 \%$ goat serum and $0.2 \%$ Triton X-100 in PBS. Myelin was visualized with a rat anti-myelin basic protein (MBP) antibody (Chemicon), followed by incubation of secondary antibodies. SCs were visualized with a rabbit anti-S100 $\beta$ antibody (DakoCytomation, High Wycombe, UK) and DRG neurons with a mouse antineurofilament antibody (American Type Culture Collection, Manassas, VA). myc-tagged BDNF was detected with a mouse anti-myc antibody (Santa Cruz Biotechnology, Santa Cruz, CA). Cellular nuclei were examined with 4',6'-diamidino-2-phenylindole (DAPI).

In situ hybridization. In situ hybridization was performed as described previously (Frantz et al., 1994). SC/DRG cocultures were grown on chamber slides and fixed in $4 \%$ paraformaldehyde for $15 \mathrm{~min}$. Slides were rinsed in PBS, dehydrated, and stored at $-80^{\circ} \mathrm{C}$. ${ }^{35}$ S-labeled $b d n f$ antisense and sense riboprobes were transcribed from linearized pGEMT plasmid containing $b d n f \mathrm{cDNA}$. Briefly, slides were pretreated with proteinase $\mathrm{K}(3.7 \mu \mathrm{g} / \mathrm{ml})$, digestion, and acetylation. After overnight hybridization at $60^{\circ} \mathrm{C}$, slides were treated with ribonuclease $\mathrm{A}(5 \mu \mathrm{g} / \mathrm{ml})$ at $37^{\circ} \mathrm{C}$. After a high-stringency wash in $0.1 \times \mathrm{SSC}$ at $60^{\circ} \mathrm{C}$, slides were dehydrated in an ethanol and xylene gradient and thoroughly air dried. Slides were then dipped in Eastman Kodak (Rochester, NY) NTB2 autoradiographic emulsion and exposed for 3 weeks before developing. After development, slides were counterstained with cresyl violet and visualized.

Adenoviral transduction of DRGs at L4/L5 in postnatal rats. mycBDNF/green fluorescent protein (GFP) or GFP virus $(2 \mu \mathrm{l})$ were injected into the ipsilateral or contralateral DRGs of 7-d-old Sprague Dawley rats, respectively. Briefly, rats were anesthetized with xylazine/ketamine. The dorsal surface of L4 and L5 DRGs were exposed by performing a double laminectomy, and virus was injected into the DRG at a rate of $\sim 1 \mu \mathrm{l} / \mathrm{min}$ using a $10 \mathrm{ml}$ Hamilton microsyringe fitted with a sterile pulled glass micropipette by inserting the tip into the ganglion. Muscle layers were sutured, and the skin was secured with wound clips. The rats were placed on soft bedding on a warming blanket held at $37^{\circ} \mathrm{C}$ for $3 \mathrm{~h}$ after surgery. Seven days later, rats were killed and perfused transcardially with $4 \%$ paraformaldehyde in $0.1 \mathrm{M}$ PBS, $\mathrm{pH}$ 7.4. DRGs and sciatic nerves were isolated and prepared for immunohistochemistry as described previously (Glatzel et al., 2000).

\section{Results \\ BDNF expression is localized to DRG neurons and is developmentally regulated}

The expression of BDNF was initially examined using competitive RT-PCR on purified SCs, DRG neurons, and SC/DRG cocultures. The coculture system is instrumental for the observation of various stages preceding, during, and after active myelination. BDNF mRNA levels were normalized and expressed as a percentage of the ribosomal protein L19 (internal control) from SC/DRG cocultures at various time points throughout myelination (Fig. $1 A)$. An increase in BDNF mRNA expression was observed shortly after purified SCs were seeded onto DRG neurons. This elevated expression was followed by a gradual decrease in BDNF mRNA throughout active myelination. Additionally, the expression of BDNF protein secreted by purified SCs, DRG neurons, and cocultures was also measured by ELISA. Consistent with the 
A.

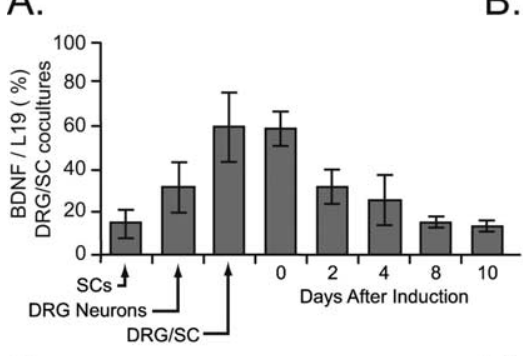

C.

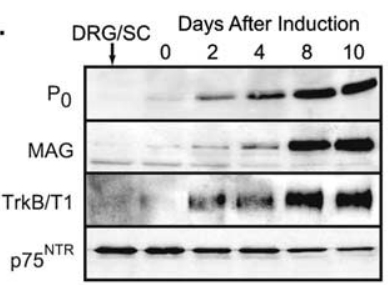

E.

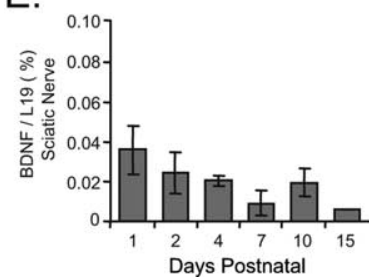

F.

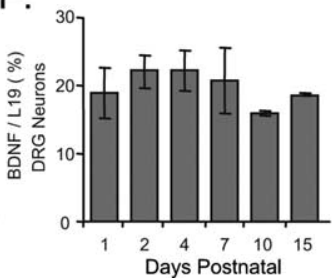

G.

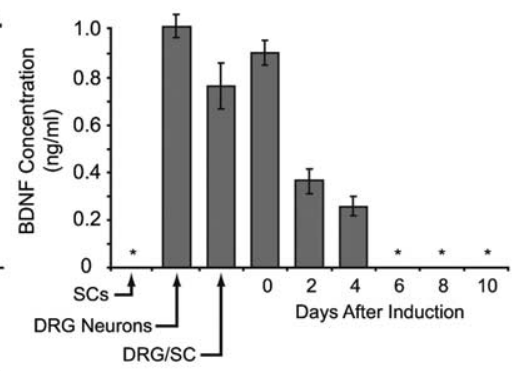

D.

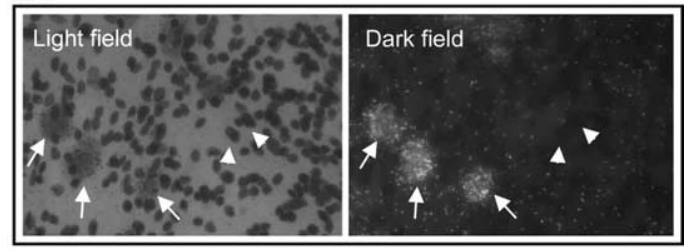

Days Postnatal

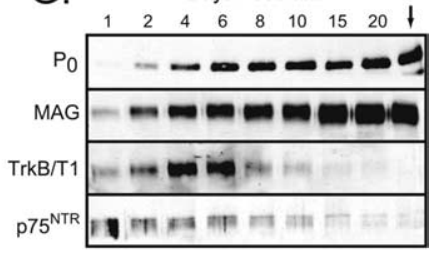

Figure 1. Expression and localization of BDNF during development. $\boldsymbol{A}$, mRNA was extracted from SCs, DRG neurons, and premyelinating $\mathrm{DRG} / \mathrm{SC}$ cocultures at the indicated days after induction of myelination with ascorbic acid. BDNF mRNA levels were analyzed by competitive RT-PCR and expressed as a percentage of the $L 19$ ribosomal protein mRNA. $\boldsymbol{B}$, Conditioned medium was collected from purified SCS, DRG neurons, and premyelinating and myelinating cocultures and analyzed by ELISA. The medium from the SCs and DRG neurons was collected throughout the entire length of the experiment. BDNF levels below detection limit are indicated by asterisks. Values represent the mean \pm SD concentration of BDNF from three separate cocultures, each measured in triplicate. $\boldsymbol{C}$, Immunoblot analysis was performed for $P_{0}, M A G, T r k B / T 1$, and $p 75^{N T R}$ from parallel cocultures. $\boldsymbol{D}$, Localization of BDNF mRNA was accomplished through the use of radioactive in situ hybridization. Premyelinating cocultures were counterstained with cresyl violet to visualize the SCs (arrowheads) and DRG neuronal cell bodies (arrows) under bright-field microscopy (left). Visualization of the probe was accomplished by autoradiography using liquid emulsion and dark-field microscopy (right). $\boldsymbol{E}$, $\boldsymbol{F}$, mRNA transcripts isolated from rat sciatic nerve and L4/L5 DRG were subjected to competitive RT-PCR to measure temporal expression levels of endogenous BDNF. Sciatic nerve and DRG samples were taken from postnatal rats as indicated. $\mathbf{G}$, Immunoblot analysis was performed for $\mathrm{P}_{0}, \mathrm{MAG}, \mathrm{TrkB} / \mathrm{T} 1$, and $\mathrm{p} 75^{\mathrm{NTR}}$ from rat sciatic nerve extracts at the indicated postnatal days. bright-field microscopy, a counterstain with cresyl violet revealed SCs occupying the field (Fig. 1D, arrowheads, left), but they accounted for little to none of the radioactivity present.

It must be noted that BDNF transcript is detected in purified SCs by RT-PCR and not by in situ hybridization or ELISA. This discrepancy is very likely attributed to differences between purified cells and cocultures. In an attempt to determine the expression of BDNF in vivo, rat sciatic nerves and the L4/L5 DRGs were examined using competitive RT-PCR. The mRNA from sciatic nerves and L4/L5 DRGs were extracted at various time points during postnatal development. BDNF mRNA levels in the sciatic nerve were found to be negligible as a percentage of the internal control and significantly lower than in the DRG (Fig. 1E,F). BDNF transcripts were $\sim 1000$-fold higher in the DRG when compared with sciatic nerves. Additionally, the expression of $\mathrm{P}_{0}, \mathrm{MAG}, \operatorname{TrkB} / \mathrm{T} 1$, and p $75^{\text {NTR }}$ were analyzed by immunoblot analysis and were consistent with the expression in the cocultures. The similarity in the temporal expression of TrkB/T1 and the initiation of myelination seemed to suggest a common developmental mechanism found both in vitro and in vivo (Fig. $1 G$ ). Together, our results demonstrate that the sensory neurons of the DRG are a major source of BDNF during postnatal development. The expression of BDNF is initially elevated before myelination and decreases dramatically after the onset of myelination. BDNF expression is controlled in part by transcriptional regulation and the increased expression of TrkB/T1 on SCs.
mRNA expression profile, purified DRG neurons cultured alone secreted significant amounts of BDNF (Fig. $1 B$ ). Although BDNF transcript was detected in purified SCs by RT-PCR, BDNF protein could not be detected by ELISA. Premyelinating cocultures secreted the highest amount of BDNF, decreasing throughout the course of active myelination. After $6 \mathrm{~d}$ of induction with ascorbic acid, BDNF levels were undetectable in the coculture medium $(\leq 0.02 \mathrm{ng} / \mathrm{ml})$. However, the BDNF mRNA levels suggest a gradual decrease in BDNF expression even at these later time points in the cocultures. Immunoblot analysis of parallel coculture lysates revealed a possible alternative regulatory mechanism for BDNF with the induction of the TrkB/T1 receptor on SCs. This induction coincides with the decrease in the level of secreted BDNF (Fig. 1B). This is consistent with previous findings that illustrate a dramatic decrease in secreted BDNF levels during active myelination, indicative of BDNF depletion by TrkB/T1 (Cosgaya et al., 2002). The expression of the myelin proteins $P_{0}$ and MAG indicate that the initiation of myelination occurs between 2 and $4 \mathrm{~d}$ after induction. To determine the localization and production of BDNF, premyelinating cocultures were analyzed for BDNF mRNA using in situ hybridization. BDNF transcripts were primarily localized in DRG neurons as evidenced by intense radioactive labeling of the cell soma (Fig. $1 D$, arrows, right). Under
Endogenous BDNF is transported in anterograde fashion and secreted along axons

To determine the mechanism of transport and secretion of endogenous BDNF, multicompartment Campenot chambers were used to physically separate the neuronal cell bodies from their axons and nerve terminals. As illustrated in Figure $2 A$, DRG neuronal cell bodies (soma) were typically cultured in the side compartment, whereas axons were directed to grow underneath the barriers and into the proximal and distal compartments. As a result, the middle compartment contained mainly axons, whereas the distal compartment contained axons and their terminals. DRG neurons were cultured for 2-3 weeks within the chambers to allow for growth and purification. The integrity of the barriers was continuously tested to eliminate the possibility of soluble factors flowing between compartments. This was achieved by quantitatively monitoring the ability of phenol red in the somal compartment to diffuse to the phenol red-free axonal compartments. Medium collected every $3 \mathrm{~d}$ was measured for the absorption of phenol red at $560 \mathrm{~nm}$, with any absorbance from the axonal compartments indicating leakage (Fig. 2C). Only those chambers that displayed both significant axon growth into the second and third compartments and had no signs of leakage were further analyzed. Medium taken from the somal and axonal 


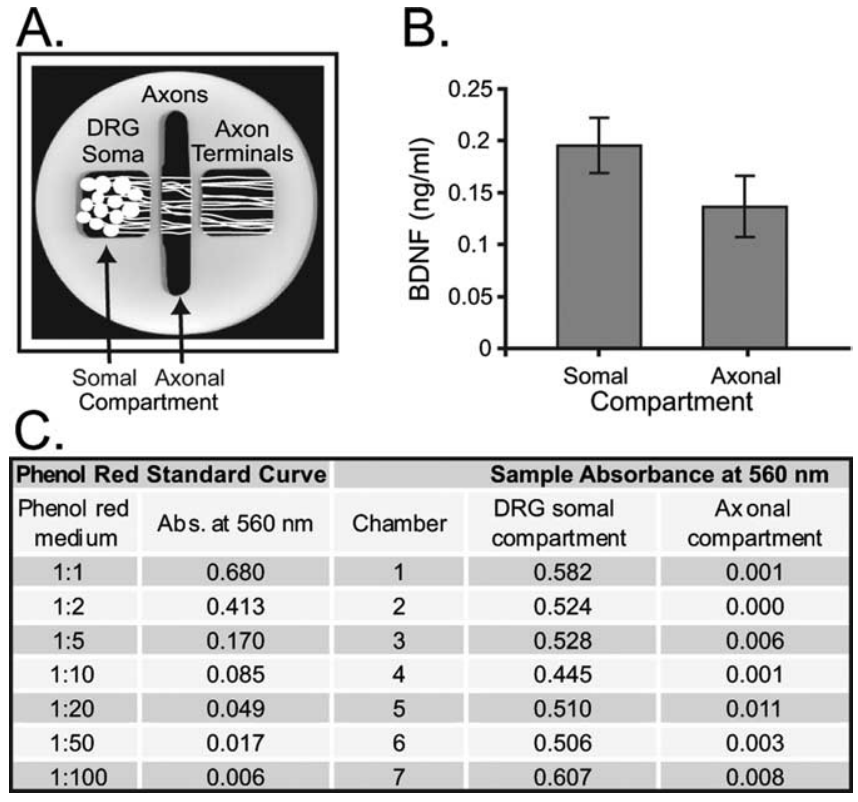

Figure 2. Endogenous BDNF is transported and secreted from DRG neuronal axons. $\boldsymbol{A}, \mathrm{DRG}$ neurons cultured in Campenot chambers were established as illustrated. The center compartment contains axons, whereas the distal compartment contains both axons and nerve terminals. $\boldsymbol{B}$, Conditioned medium taken from the DRG somal and middle axonal compartments were analyzed by ELISA for BDNF. Values represent the mean \pm SD concentration of BDNF from seven separate chambers. $\boldsymbol{C}$, The integrity of the chamber barriers was continuously tested from seven different chambers to eliminate the possibility of soluble factors flowing between compartments. This was achieved by quantitatively monitoring the ability of phenol red in the somal compartment to diffuse to the phenol red-free axonal compartments.

compartments were assayed for BDNF by ELISA (Fig. $2 B$ ). In addition to significant amounts of BDNF secreted in the DRG somal compartment, a comparable amount was detected in the axonal compartment. On average, $\sim 0.15 \mathrm{ng} / \mathrm{ml}$ BDNF was detected in either compartment. The BDNF concentrations between the two compartments were not significantly different as determined by Student's $t$ test. Results from this assay indicate that BDNF is not only secreted from neuronal cell bodies but is also anterogradely transported and secreted along the surface of the axon.

\section{Adenoviral-mediated overexpression of BDNF in DRG neurons enhances SC myelination}

Myc-tagged BDNF adenoviral constructs, which were coexpressed with GFP, were designed to overexpress BDNF in DRG neuron cultures. Approximately 1 week after infection, DRG neurons were fixed and stained with an anti-myc antibody (Fig. $3 A b, A d)$. Parallel neuronal cultures infected with a GFP construct were similarly immunostained (Fig. $3 B b, B d$ ). Adenoviralmediated infection of primary neuronal cultures was achieved at high efficiency ( $\geq 80 \%$ ). All experimental cultures were extensively populated with GFP-expressing axons. Neurons that were infected with the myc-BDNF construct also showed distinct myc immunoreactivity in cell bodies and along axonal tracts (Fig. $3 A b, A d)$. Minimal myc immunoreactivity was seen in cultures infected with the GFP construct alone (Fig. $3 B b, B d$ ). Approximately 1 week after infection, DRG neuronal cultures were seeded with purified SCs to gauge the effects of BDNF overexpression on myelin formation. BDNF expression was examined by ELISA (Fig. 3C). Secreted BDNF levels from the myc-BDNFinfected DRG neuronal cultures were $\sim 70$-fold greater than endogenous BDNF levels from the GFP-infected cultures (Fig. 3D).
A similar increase was observed in the SC/DRG neuronal cocultures during premyelinating periods. Surprisingly, a dramatic decrease in BDNF expression was detected even in the myc-BDNFinfected cultures after $4 \mathrm{~d}$ of induction, in a similar manner to the GFP-infected cocultures (Fig. 3C,D). After $10 \mathrm{~d}$ of induction, cocultures were immunostained with an anti-MBP antibody to visualize the extent of myelin formation in the infected cocultures. Myelination of the myc-BDNF-infected neurons was approximately fivefold greater when counting the percentage of myelinating Schwann cells per field compared with GFP-infected cultures (Fig. 3E-G).

\section{myc-BDNF is transported in an anterograde manner and secreted along DRG axons to promote Schwann cell myelination}

Campenot chambers were also used to determine the method of BDNF delivery to SCs. DRG neuronal cell bodies cultured in Campenot chambers were infected with either the myc-BDNF or the GFP adenoviral construct as described previously. The adenovirus was removed after overnight infection, and the neurons were cultured for 1 week before SCs were seeded into the center axonal compartment (Fig. 4A). Three days after seeding, the cocultures were stained with an anti-myc antibody (Fig. $4 B$ ). In the cocultures with myc-BDNF-infected neurons, myc immunoreactivity (red) was seen in almost all SCs that had aligned or were in direct contact with GFP-positive axons. This was indicative of secretion and transfer of myc-BDNF from the axon to the SC. Myc immunoreactivity was minimal in control cocultures with GFP-only infected neurons. On closer inspection of myc-positive SCs, transfer of myc-BDNF was clearly evident by intense myc immunoreactivity in the SC body (Fig. 4C). SCs were clearly identified by $\mathrm{S} 100 \beta$ immunoreactivity (blue). myc-positive SCs were not GFP positive from aberrant adenoviral infection. In parallel cocultures, medium from the DRG somal and axonal compartments were assayed for BDNF secretion $8 \mathrm{~d}$ after induction of myelination. Both compartments contained high levels of secreted BDNF in the infected neurons (Fig. 4D). Approximately $100 \mathrm{ng} / \mathrm{ml}$ BDNF was detected in the somal compartments, whereas up to $20 \mathrm{ng} / \mathrm{ml} \mathrm{BDNF}$ was detected in the axonal compartments. Comparatively lower amounts of endogenous BDNF $(0.2 \mathrm{ng} / \mathrm{ml})$ were detected in the DRG somal compartments of the GFP-infected neurons. Virtually no BDNF was detected in the axonal compartments, illustrating once again the dramatic decrease in BDNF protein levels during active myelination (Chan et al., 2001). The infected cocultures were then analyzed for myelin formation in the center compartments by MBP immunostaining and quantification. Cocultures infected with the myc-BDNF adenovirus had threefold more myelin internodes than the GFPinfected cocultures (Fig. 4E).

\section{Discussion}

Various mechanisms have been proposed that describe the expression and transport of neurotrophins in the nervous system. Traditionally, neurotrophins have been described as "targetderived" signaling molecules, influencing the growth and survival of innervating neurons (Lessmann et al., 2003). This suggests that target tissues express and secrete neurotrophins, whereby they are internalized by axon terminals and activate signaling pathways in neuronal cell bodies after retrograde transport (Howe and Mobley, 2005; Zweifel et al., 2005). In certain cases, neurotrophins can also be expressed by neurons and act by anterograde transport mechanisms (Zhou and Rush, 1996; Tonra et al., 1998). Double ligation of rat sciatic nerves results in the ac- 
A.
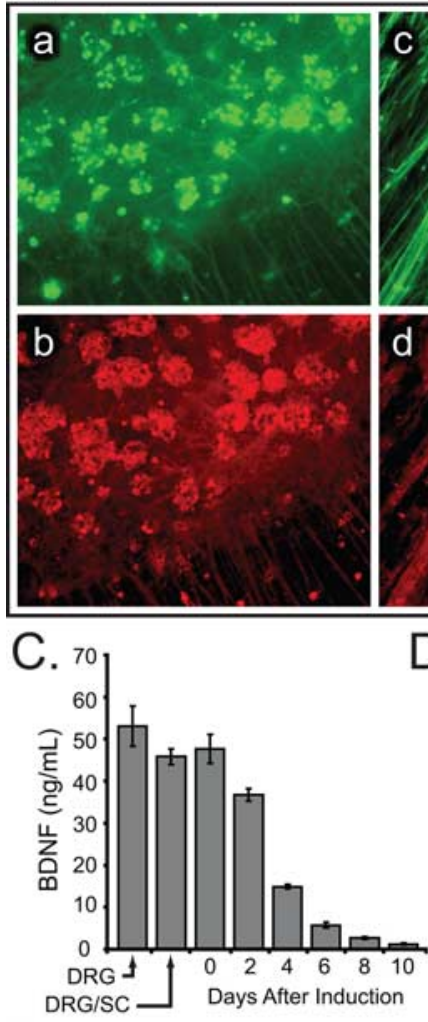

$\mathrm{F}$.
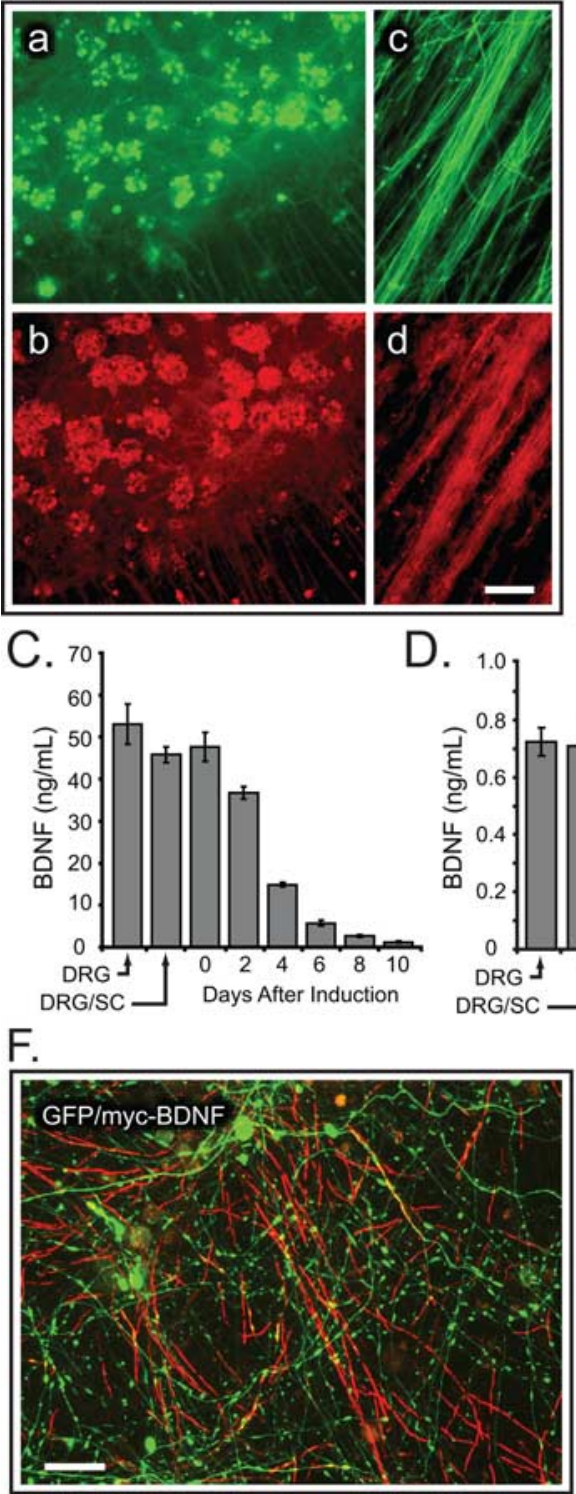

D
B.

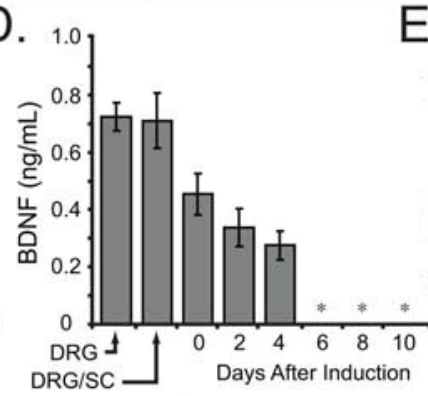

G.

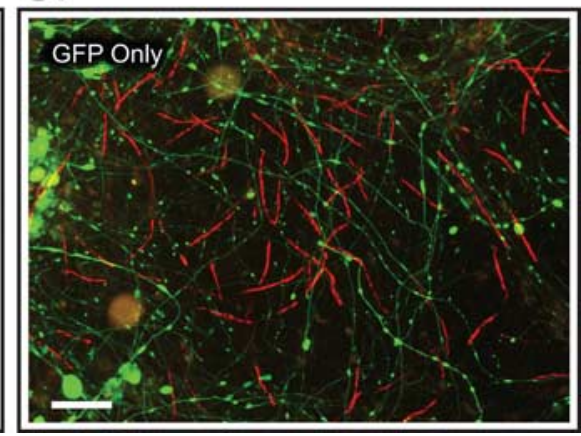

Figure 3. Overexpression of BDNF in SC/DRG cocultures enhances myelin formation. $\boldsymbol{A}, \boldsymbol{B}$, Purified DRG neurons were infected with a myc-BDNF or GFP adenoviral construct for $12 \mathrm{~h}$. Seven days after initial infection, the neurons were visualized for GFP fluorescence ( $\boldsymbol{A a}, \boldsymbol{A c}, \boldsymbol{B a}, \boldsymbol{B C})$ and also immunostained with an anti-myc antibody $(\boldsymbol{A b}, \boldsymbol{A d}, \boldsymbol{B} \boldsymbol{b}, \boldsymbol{B} \boldsymbol{d})$. Scale bar, $50 \mu \mathrm{m}$. $\boldsymbol{C}, \boldsymbol{D}, \mathrm{DRG}$ neuron cultures infected with a myc-BDNF $(\boldsymbol{C})$ or GFP $(\boldsymbol{D})$ were seeded with purified SCs and analyzed for BDNF at various time points during myelin formation. BDNF levels below detection limit are indicated by asterisks. Values represent the mean \pm SD concentration of BDNF from three separate cocultures, each measured in triplicate by ELISA. E-G, Myc-BDNF and GFP cocultures were stained for MBP (red) to measure the extent of myelination after $10 \mathrm{~d}$ of induction. An approximate fivefold increase in the percentage of myelinating Schwann cells was observed in the myc-BDNF-infected cocultures when compared with the GFPinfected cocultures. Scale bar, $100 \mu \mathrm{m}$. Significance was evaluated using Student's $t$ test $\left({ }^{*} p<0.005\right)$.

cumulation of BDNF both proximally and distally to the ligated segment, illustrating both anterograde and retrograde transport, respectively (Zhou and Rush, 1996). It has also been suggested that local protein synthesis can contribute to the axonal protein pool. Although it is possible that BDNF is locally synthesized in the axons, studies illustrate an upregulation in axonal protein synthesis only after nerve transection (Zheng et al., 2001). Additionally, in intact nerves, ribosomes are typically found in high abundance at the initial axonal segments near the DRG (Pannese and Ledda, 1991). Concurrent to the results shown in this study, in vivo experiments demonstrate that the initial overexpression of myc-BDNF/GFP in L4 and L5 DRG neurons can later be detected in corresponding axons of the sciatic nerve (supplemental Fig. 1, available at www.jneurosci.org as supplemental material). The expression of BDNF in the sciatic nerve provides additional support for its anterograde transport from the cell body. Together, sensory neurons produce and transport BDNF in an anterograde manner to axon terminals in which either autocrine or paracrine pathways are initiated through TrkB and/or p75 ${ }^{\text {NTR }}$. However, because SCs do not myelinate nerve terminals, could BDNF be secreted along the surface of the axon? This is an issue that has not been thoroughly examined because of a lack of functional significance.

We propose that the process of myelination requires direct signaling from the axon. Recent evidence has pointed to the importance of axonal signaling in SC myelination with the finding that axonal neuregulin-1 type III encodes for a physiological measure of axon size to regulate the thickness of the myelin sheath (Michailov et al., 2004; Taveggia et al., 2005). We demonstrate that the anterograde transport and secretion of BDNF from the neuronal axon enhances myelin formation by SCs in vitro. Admittedly, our data does not address the specific activation of $\mathrm{p} 75^{\mathrm{NTR}}$ on SCs and that BDNF may potentially activate $\mathrm{p} 75^{\mathrm{NTR}}$ on sensory axons. However, our data clearly demonstrate that BDNF secreted by DRGs is taken up by SCs. This is consistent with our recent finding that the polarity protein Par-3 is enriched at the axon/SC interface and actively binds and recruits $\mathrm{p} 75^{\mathrm{NTR}}$ in response to BDNF (Chan et al., 2006).

Previously, a number of different strategies involving myelin repair have been reported, including the transplantation of exogenous cell types that are often engineered to overexpress neurotrophins or other growth factors. For example, NT3 and BDNF transduced macaque SCs were grafted into demyelinated spinal cords of nude mice (Girard et al., 2005). The secreted neurotrophins successfully promoted clinical recovery, enhanced oligodendrocyte precursor cell and SC differentiation, and reduced astrogliosis (Girard et al., 2005; Tepavcevic and Blakemore, 2005). In other studies, engineered, native fibroblasts were used to deliver a constant flow of neurotrophins to injury sites. Primary rat fibroblasts were infected with a construct encoding NT3 and grafted into lesioned spinal cords of adult rats. Increased axonal outgrowth was reported in the treated spinal cords, but only partial functional recovery was obtained (Grill et al., 1997). Similarly, our data hopes to contribute to more efficient and novel therapeutic strategies to achieve functional myelin repair. Our studies demonstrate that sensory neurons of the DRG are a major source of BDNF during development and that increasing BDNF expression in neurons significantly 
A.

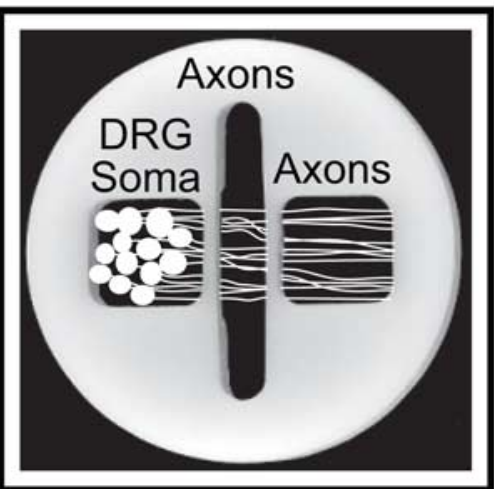

C.
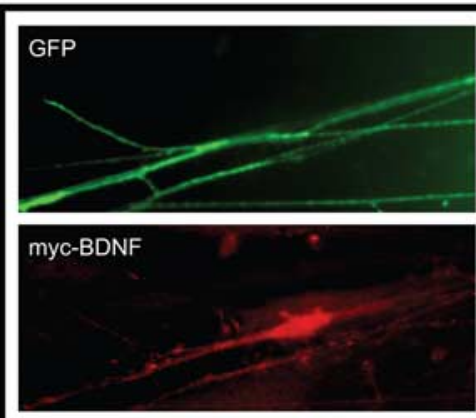

GFP/myc-BDNF Merge

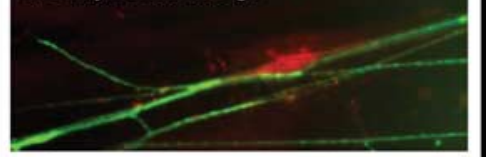

$S 100 \beta$

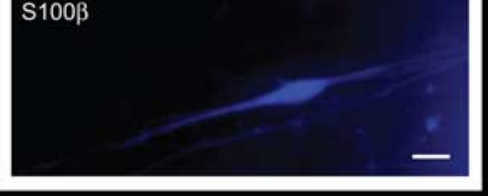

B

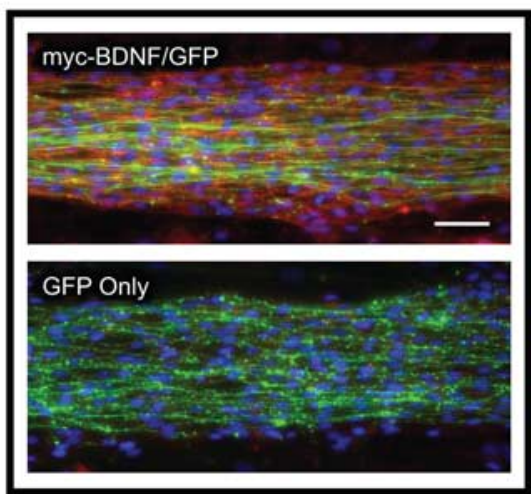

D.

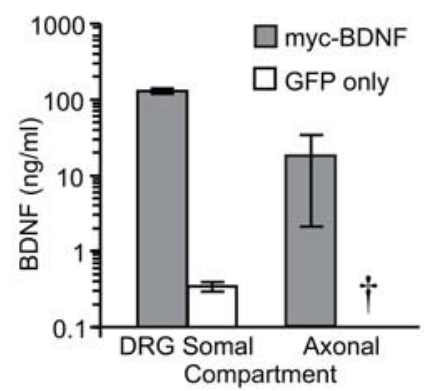

E.

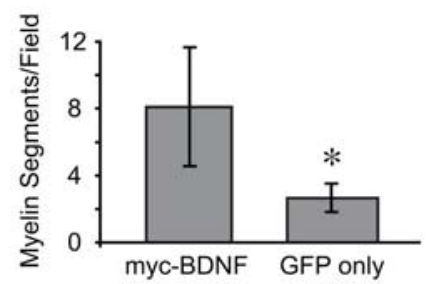

Figure 4. myc-BDNF is transported in an anterograde manner and secreted along DRG axons to promote Schwann cell myelination. $A, D R G$ neurons cultured in Campenot chambers were established as illustrated. $B$, DRG neurons were infected with either the myc-BDNF/GFP or the GFP-only adenoviral construct in the somal compartment. Seven days after initial infection, purified SCS were seeded onto the axons in the center compartment. GFP fluorescence (green) and myc immunoreactivity (red) were analyzed in the center compartment $3 \mathrm{~d}$ after seeding. Schwann cell nuclei were stained with DAPI (blue). Scale bar, $50 \mu \mathrm{m}$ (for both panels). C, Similar cocultures were analyzed for GFP fluorescence (green) and myc immunoreactivity (red) $7 \mathrm{~d}$ after seeding. S100 $\beta$ immunoreactivity (blue) was used to identify SCs. Scale bar, $10 \mu \mathrm{m}$ (for all panels). D, Eight days after induction of myelination, conditioned medium from the somal and axonal compartments of parallel cocultures were assayed for BDNF. BDNF concentrations were presented along a logarithmic scale. Endogenous BDNF from the GFP-infected neurons were below the detection limit in the axonal compartment (†). $E$, Myelin segments were also quantified using MBP staining in the center compartment to assess the extent of myelin formation in the infected cocultures. All values represent the mean \pm SD from separate experiments. Significance was evaluated using Student's $t$ test $\left({ }^{*} p<0.005\right)$.

enhances the number of SC myelin internodes. Although the experiments presented here were performed in vitro, it is our hope that these findings may translate to in vivo models, with the potential therapeutic role of overexpressing BDNF in sensory neurons to promote remyelination after nerve injury or demyelinating disease.

\section{References}

Barakat-Walter I (1996) Brain-derived neurotrophic factor-like immunoreactivity is localized mainly in small sensory neurons of rat dorsal root ganglia. J Neurosci Methods 68:281-288.

Campenot RB (1992) Compartmented culture analysis of nerve growth. In: Cell-cell interactions: a practical approach (Snevenson BR, Gallin WJ, Paul DL, eds), pp 275-299. New York: Oxford UP.
Chan JR, Cosgaya JM, Wu YJ, Shooter EM (2001) Neurotrophins are key mediators of the myelination program in the peripheral nervous system. Proc Natl Acad Sci USA 98:14661-14668.

Chan JR, Watkins TA, Cosgaya JM, Zhang C, Chen L, Reichardt LF, Shooter EM, Barres BA (2004) NGF controls axonal receptivity to myelination by Schwann cells or oligodendrocytes. Neuron 43:183-191.

Chan JR, Jolicoeur C, Yamauchi J, Elliott J, Fawcett JP, Ng BK, Cayouette MC (2006) The polarity protein Par-3 directly interacts with p75NTR to regulate myelination. Science 314: 832-836.

Cosgaya JM, Chan JR, Shooter EM (2002) The neurotrophin receptor $\mathrm{p} 75 \mathrm{NTR}$ as a positive modulator of myelination. Science 298: $1245-1248$.

Esper RM, Loeb JA (2004) Rapid axoglial signaling mediated by neuregulin and neurotrophic factors. J Neurosci 24:6218-6227.

Frantz GD, Weimann JM, Levin ME, McConnell SK (1994) Otx1 and Otx2 define layers and regions in developing cerebral cortex and cerebellum. J Neurosci 14:5725-5740.

Friedman HC, Jelsma TN, Bray GM, Aguayo AJ (1996) A distinct pattern of trophic factor expression in myelin-deficient nerves of Trembler mice: implications for trophic support by Schwann cells. J Neurosci 16:5344-5350.

Friedman HC, Aguayo AJ, Bray GM (1999) Trophic factors in neuron-Schwann cell interactions. Ann NY Acad Sci 883:427-438.

Girard C, Bemelmans AP, Dufour N, Mallet J, Bachelin C, Nait-Oumesmar B, Baron-Van Evercooren A, Lachapelle F (2005) Grafts of brain-derived neurotrophic factor and neurotrophin 3-transduced primate Schwann cells lead to functional recovery of the demyelinated mouse spinal cord. J Neurosci 25:7924-7933.

Glatzel M, Flechsig E, Navarro B, Klein MA, Paterna JC, Bueler H, Aguzzi A (2000) Adenoviral and adeno-associated viral transfer of genes to the peripheral nervous system. Proc Natl Acad Sci USA 97:442-447.

Graham FL, Prevec L (1995) Methods for construction of adenovirus vectors. Mol Biotechnol 3:207-220.

Grill R, Murai K, Blesch A, Gage FH, Tuszynski MH (1997) Cellular delivery of neurotrophin-3 promotes corticospinal axonal growth and partial functional recovery after spinal cord injury. J Neurosci 17:5560-5572.

He TC, Zhou S, da Costa LT, Yu J, Kinzler KW, Vogelstein B (1998) A simplified system for generating recombinant adenoviruses. Proc Natl Acad Sci USA 95:2509-2514.

Hempstead BL (2005) Coupling neurotrophins to cell migration through selective guanine nucleotide exchange factor activation. Proc Natl Acad Sci USA 102:5645-5646.

Howe CL, Mobley WC (2005) Long-distance retrograde neurotrophic signaling. Curr Opin Neurobiol 15:40-48.

Huang EJ, Reichardt LF (2001) Neurotrophins: roles in neuronal development and function. Annu Rev Neurosci 24:677-736.

Kleitman N, Wood PM, Bunge RP (1998) Tissue culture methods for the study of myelination. In: Culturing nerve cells, Ed 2 (Banker G, Goslin K, eds), pp 545-594. Cambridge, MA: MIT.

Kruttgen A, Moller JC, Heymach Jr JV, Shooter EM (1998) Neurotrophins induce release of neurotrophins by the regulated secretory pathway. Proc Natl Acad Sci USA 95:9614-9619. 
Lessmann V, Gottmann K, Malcangio M (2003) Neurotrophin secretion: current facts and future prospects. Prog Neurobiol 69:341-374.

Michailov GV, Sereda MW, Brinkmann BG, Fischer TM, Haug B, Birchmeier C, Role L, Lai C, Schwab MH, Nave KA (2004) Axonal neuregulin-1 regulates myelin sheath thickness. Science 304:700-703.

Pannese E, Ledda M (1991) Ribosomes in myelinated axons of the rabbit spinal ganglion neurons. J Submicrosc Cytol Pathol 23:33-38.

Taveggia C, Zanazzi G, Petrylak A, Yano H, Rosenbluth J, Einheber S, Xu X, Esper RM, Loeb JA, Shrager P, Chao MV, Falls DL, Role L, Salzer JL (2005) Neuregulin-1 type III determines the ensheathment fate of axons. Neuron 47:681-694.

Tepavcevic V, Blakemore WF (2005) Glial grafting for demyelinating disease. Philos Trans R Soc Lond B Biol Sci 360:1775-1795.

Tonra JR (1999) Classical and novel directions in neurotrophin transport and research: anterograde transport of brain-derived neurotrophic factor by sensory neurons. Microsc Res Tech 45:225-232.

Tonra JR, Curtis R, Wong V, Cliffer KD, Park JS, Timmes A, Nguyen T, Lindsay RM, Acheson A, DiStefano PS (1998) Axotomy upregulates the anterograde transport and expression of brain-derived neurotrophic factor by sensory neurons. J Neurosci 18:4374-4383.
Wu YJ, Kruttgen A, Moller JC, Shine D, Chan JR, Shooter EM, Cosgaya JM (2004) Nerve growth factor, brain-derived neurotrophic factor, and neurotrophin-3 are sorted to dense-core vesicles and released via the regulated pathway in primary rat cortical neurons. J Neurosci Res 75:825-834.

Yamauchi J, Chan JR, Shooter EM (2003) Neurotrophin 3 activation of TrkC induces Schwann cell migration through the c-Jun N-terminal kinase pathway. Proc Natl Acad Sci USA 100:14421-14426.

Yamauchi J, Chan JR, Shooter EM (2004) Neurotrophins regulate Schwann cell migration by activating divergent signaling pathways dependent on Rho GTPases. Proc Natl Acad Sci USA 101:8774-8779.

Zheng JQ, Kelly TK, Chang B, Ryazantsev S, Rajasekaran AK, Martin KC, Twiss JL (2001) A functional role for intra-axonal protein synthesis during axonal regeneration from adult sensory neurons. J Neurosci 21:9291-9303.

Zhou XF, Rush RA (1996) Endogenous brain-derived neurotrophic factor is anterogradely transported in primary sensory neurons. Neuroscience 74:945-953.

Zweifel LS, Kuruvilla R, Ginty DD (2005) Functions and mechanisms of retrograde neurotrophin signalling. Nat Rev Neurosci 6:615-625. 